\title{
INFLUÊNCIA DA TEMPERATURA E VELOCIDADE DO AR NA SECAGEM DE MANJERICÃO (Ocimum basilicum L.) COM RELAÇÃO AOS TEORES DE ÓLEOS ESSENCIAIS E DE LINALOL ${ }^{1}$
}

\author{
Influence of drying temperature and air velocity related to essential oil and linalol \\ contents of the basil (Ocimum basilicum L.)
}

\author{
Rilvaynia Dantas Soares ${ }^{2}$, Modesto Antonio Chaves ${ }^{3}$, Arienilmar Araujo Lopes da Silva ${ }^{4}$, \\ Marcondes Viana da Silva ${ }^{5}$, Betânia dos Santos Souza ${ }^{6}$
}

\begin{abstract}
RESUMO
Neste trabalho, objetivou-se estudar a influência de quatro temperaturas de ar de secagem $\left(40,50,60 \mathrm{e} 70{ }^{\circ} \mathrm{C}\right)$, em camada fina, e duas velocidades do ar $(0,9$ e $1,9 \mathrm{~m} / \mathrm{s})$ sobre o teor de linalol do manjericão (Ocimum basilicum $\mathrm{L}$ ). Utilizou-se um secador de bandejas especialmente desenvolvido, no laboratório de Secagem da UESB, Campus de Itapetinga-BA. Foram feitas quatro repetições, com $100 \mathrm{~g}$ de folhas frescas em cada uma das cinco bandejas da câmara de secagem. Na extração do óleo essencial, utilizou-se um sistema de destilação por arraste a vapor e a análise do óleo essencial foi realizada em um cromatógrafo conectado a um e espectrômetro de massa (CG-MS). Os maiores rendimentos de óleos essenciais de manjericão foram obtidos no processo de secagem com temperatura do ar igual a $40^{\circ} \mathrm{C}$ e $1,9 \mathrm{~m} / \mathrm{s}$ de velocidade do ar. Os maiores rendimentos de linalol foram obtidos com temperatura do ar de secagem na faixa de 50 a $60{ }^{\circ} \mathrm{C}$ e $1,9 \mathrm{~m} / \mathrm{s}$ de velocidade do ar (2,23 e 2,47 ppm, respectivamente). Por análise de regressão, estimou-se que a temperatura de $54,4^{\circ} \mathrm{C}$ e a velocidade de $1,9 \mathrm{~m} / \mathrm{s}$ forneceriam o maior rendimento de linalol. Concluiu-se que a composição química do óleo essencial do manjericão é afetada tanto pela temperatura como pela velocidade do ar de secagem.
\end{abstract}

Termos para indexação: Secagem, plantas medicinais, rendimento de óleo.

\section{ABSTRACT}

This work aimed to study the influence of four thin layer drying temperatures $\left(40,50,60\right.$ and $\left.70{ }^{\circ} \mathrm{C}\right)$ and two air velocities $(0.9$ and $1.9 \mathrm{~m} / \mathrm{s}$ ) in the content of linalol of the Basil (Ocimum basilicum L.). It was used a tray dryer specially built for this experiment in the drying laboratory of the UESB at the "Campus de Itapetinga-Ba". There were made four repetitions with $100 \mathrm{~g}$ of fresh leaves in each one of the five trays in the dry chamber In the extraction of the essential oil, a vapor hauling system was used and the oil analysis was made in a gas chromatographer that was connected to a mass spectrometer (CG-MS). The greatest extracted profits of basil's essential oil were obtained in the drying process with $40^{\circ} \mathrm{C}$ of air temperature and $1.9 \mathrm{~m} / \mathrm{s}$ of air velocity. The greatest profit of linalol was obtained with the air drying temperature in the range of $50-60^{\circ} \mathrm{C}$ and $1.9 \mathrm{~m} / \mathrm{s}$ of air velocity $(2.23$ and $2.47 \mathrm{ppm}$, respectively). It was estimated, by regression analysis, that the air drying temperature of $54.4^{\circ} \mathrm{C}$ and the air flow of $1.9 \mathrm{~m} / \mathrm{s}$ would provide the greatest profit of linalol. It was concluded that the chemical composition of the basil was affected by both drying air temperature and velocity.

Index terms: Drying, medicinal plants, oil yelds.

(Recebido em 5 de junho de 2006 e aprovado em 15 de março de 2007)

\section{INTRODUÇÃO}

O Ocimum basilicum L. é originário do norte da Índia e age como estimulante digestivo, antiespasmódico, antisséptico e béquico (MARTINS et al., 1994). Pertencentes à família Lamiaceae são plantas conhecidas popularmente como manjericão ou alfavaca (JORGE et al., 1992; KAMADA et al., 1999).

As espécies mais conhecidas apresentam como constituintes majoritários em seu óleo essencial o metilchavicol, eugenol, linalol, 1,8-cineol (BARITAUX et al., 1992), cinamato de metila (PEREZ et al., 1995), geraniol (CHARLES \& SIMON, 1990) e timol (NTEZURUBANZA et al., 1984).

Limitadas informações estão disponíveis na literatura relativas aos efeitos da temperatura e da umidade do ar de secagem sobre o teor dos princípios ativos de plantas medicinais durante o pré-processamento. Segundo Martins (2000), é possível que o menor conteúdo de água

\footnotetext{
${ }^{1}$ Extraído da dissertação de mestrado do primeiro autor.

${ }^{2}$ Mestre em Agronomia - Universidade Estadual do Sudoeste da Bahia/UESB - Praça Primavera, 40 Bairro Primavera - 45700-000 - Itapetinga, BA rilvaynia@yahoo.com.br - Bolsista AT 1 - FAPESB

${ }^{3}$ Doutor em Agronomia, Professor Titular - Departamento de Estudos Básicos e Instrumentais - Universidade Estadual do Sudoeste da Bahia/UESB Praça Primavera, 40 Bairro Primavera - 45700-000 - Itapetinga, BA - modesto@uesb.br

${ }^{4}$ Doutor em Engenharia Agrícola, Professor Adjunto - Departamento de Tecnologia Rural e Animal - Universidade Estadual do Sudoeste da Bahia/UESB Praça Primavera, 40 Bairro Primavera - 45700-000 - Itapetinga, BA - arienilmar@hotmail.com

${ }^{5}$ Doutor em Ciência e Tecnologia de Alimentos, Professor Adjunto - Departamento de Estudos Básicos e Instrumentais - Universidade Estadual do Sudoeste da Bahia/UESB - Praça Primavera, 40 Bairro Primavera - 45700-000 - Itapetinga, BA - mviana@hotmail.com

${ }^{6}$ Graduanda em Engenharia de Alimentos - Departamento de Estudos Básicos e Instrumentais - Universidade Estadual do Sudoeste da Bahia/UESB -

Praça Primavera, 40 Bairro Primavera - 45700-000 - Itapetinga, BA - bethany_701@yahoo.com.br
} 
nas folhas, após a secagem, permita que a corrente de vapor gerada no extrator possa arrastar, mais eficientemente, as substâncias voláteis armazenadas nas células, quando comparado com material verde.

Pesquisas recentes sobre secagem de plantas medicinais têm sido direcionadas para o estudo dos seguintes parâmetros: a) temperatura e velocidade do ar de secagem; b) umidade relativa do ar dentro e fora do sistema de secagem; c) temperatura do material; d) pressão estática em função da altura da camada do produto.

Existe grande variação em relação aos valores de velocidade do ar recomendados para secagem de plantas medicinais. Hansen et al. (1993) utilizaram a velocidade de $0,039 \mathrm{~m} / \mathrm{s}$, num secador de leito fixo, trabalhando com camada delgada de raspas de Taxus hicks. Na secagem de manjericão (Ocimum basilicum L.), a velocidade, utilizada por Baritaux et al. (1992), foi de 0,4 m/s. Müller \& Muhlbauer (1992), utilizaram $0,2 \mathrm{~m} / \mathrm{s}$ na secagem de folhas de sálvia (Salvia officinalis L.).

Em alguns casos, a secagem artificial resultou no maior rendimento em óleo e/ou, na maior concentração do componente ativo, como por exemplo a secagem de tomilho (Thymus vulgaris L) a $30^{\circ} \mathrm{C}$ por Venskutonis et al. (1996) onde estes descreveram uma maior concentração de bcariofileno no produto seco $(288 \mathrm{mg} / \mathrm{Kg})$ quando compararam o teor do mesmo no produto fresco $(213 \mathrm{mg} /$ $\mathrm{Kg}$ ). Venskutonis (1997), comparando a secagem de tomilho e sálvia observou um maior rendimento (29\%) na concentração de b-cariofileno no tomilho seco a $30^{\circ} \mathrm{C}$ sem que,contudo, houvesse variação no teor de timol, o que não ocorreu com a sálvia em relação ao seu componente principal.

Radünz et al. (2002) estudaram a influência da temperatura do ar de secagem sobre a composição química do óleo essencial extraído do alecrim pimenta (Lippia sidoides Cham). Concluíram que os teores de p-cimeno e timol, presentes no óleo essencial, não sofreram variações significativas em função das temperaturas de secagem empregadas, relativamente ao do controle. Entretanto, o teor de cariofileno apresentou aumento significativo quando submetido às temperaturas de 50,60 e $70^{\circ} \mathrm{C}$.

Radünz et al. (2003) avaliaram a influência da temperatura do ar de secagem sobre a quantidade de óleo essencial extraído do guaco (Mikania glomerata S.) e observaram que a utilização da temperatura igual a $55^{\circ} \mathrm{C}$, para o ar de secagem, não influenciou estatisticamente na quantidade de óleo essencial extraído.

O óleo essencial de manjericão é importado e comercializado no Brasil em pequenas quantidades, atingindo valores de U\$ 41 a U\$ 50 o kg/FOB (GEORGE, 1995, citado por TEIXEIRA et al., 2000).
Dado o valor econômico do óleo e a pequena quantidade de informação existente sobre a influência de parâmetros de secagem sobre o teor do mesmo, conduziuse este trabalho para estudar a influência de quatro temperaturas de secagem e duas vazões de ar, sobre o teor de linalol do manjericão (Ocimum basilicum L.).

\section{MATERIAL E MÉTODOS}

Os ensaios de secagem das folhas de manjericão foram realizados no Laboratório de Secagem do Módulo de Laboratórios da Universidade Estadual do Sudoeste da Bahia (UESB), Campus Juvino Oliveira em Itapetinga, durante o período de 19 a 26 de setembro de 2005 .

As plantas de Ocimum basilicum L. foram cultivadas na área experimental de agrometeorologia da UESB, localizada no município de Itapetinga, Ba, situado a latitude de $15^{\circ} 18^{\prime} \mathrm{S}$ e longitude $40^{\circ} 15^{\prime} \mathrm{W}$, temperatura média anual de $27^{\circ} \mathrm{C}$, altitude média de $268 \mathrm{~m}$ e clima, segundo a classificação de Köppen, caracterizado como Aw (quente e úmido com estação seca de inverno).

As análises físico-químicos do solo registraram níveis satisfatórios de nutrientes, sendo realizadas adubações orgânicas (esterco bovino) durante o ciclo da cultura. Utilizou-se sementes da empresa Feltrin Sementes, sendo semeadas em sementeiras no dia 13 de junho de 2005, e transplantadas quando atingiram 0,10 m de altura para canteiros previamente preparados. As mudas foram colocadas em covas de $0,15 \mathrm{~m}$ de profundidade, no espaçamento de $0,50 \mathrm{~m}$ entre plantas e $0,50 \mathrm{~m}$, entre linhas. Os tratos culturais realizados no campo foram a capina manual, irrigação por aspersão uma vez ao dia, e retirada das inflorescências durante todo o ciclo. As plantas foram tutoradas com bambu (amarradas com barbante), quando atingiram $0,60 \mathrm{~m}$ de altura.

A colheita do material foi realizada no período de 19 a 26 de setembro de 2005, das 07:00 ás 08:00 h, devido à maior concentração do óleo essencial pela manhã (MARTINS, 1998). As folhas foram selecionadas, removendo partes doentes, danificadas e/ou atacadas por insetos.

Os teores de umidade iniciais das folhas do manjericão foram determinados imediatamente após a coleta e no final do processo de secagem. Utilizou-se a metodologia recomendada pela ASAE STANDARDS (ASAE STANDARDS, 2000), para forrageiras e similares (plantas ou folhas), colocando-se $20 \mathrm{~g}$ de amostra, em três repetições, em estufa com circulação forçada do ar e temperatura de $105 \pm 2{ }^{\circ} \mathrm{C}$, durante 24 horas.

Para a secagem das folhas do manjericão foi utilizado um secador desenvolvido no Laboratório de Secagem da UESB, Campus de Itapetinga, apresentado na Figura 1. 


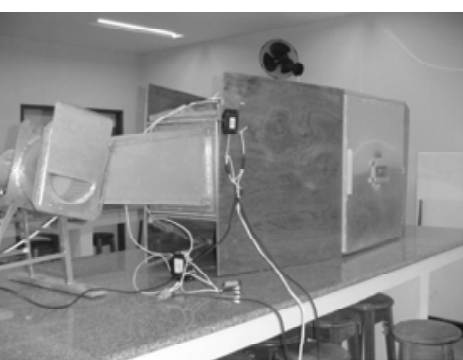

(a)

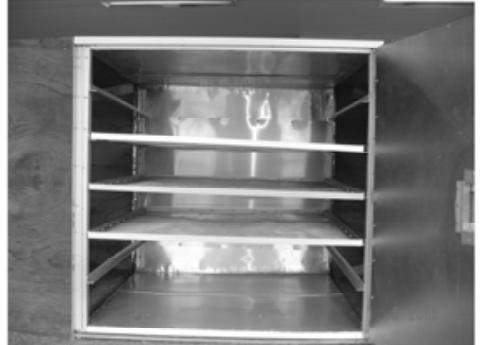

(b)

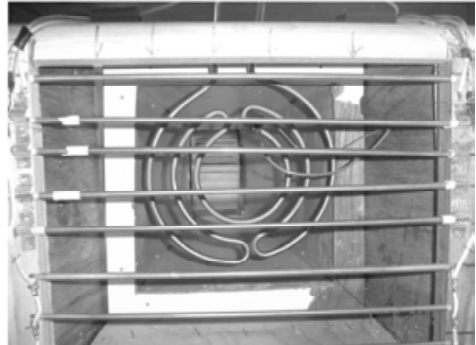

(c)

FIGURA 1 - Secador: (a) vista lateral (b) câmara de secagem (c) resistências elétricas.

Em cada uma das cinco bandejas do secador foram distribuídas, em camada fina, $100 \mathrm{~g}$ de folhas frescas. Uma pequena bandeja com uma amostra de $20 \mathrm{~g}$ foi colocada no meio da bandeja central e, pesagens desta, durante o processo de secagem, foram realizadas em intervalos de 15 minutos, em balança semi-analítica (Gehaka BG 4400, com precisão de $\pm 0,01 / 0,1 \mathrm{~g}$ ) de forma a se controlar a secagem.

Foram utilizadas quatro temperaturas de secagem $\left(40,50,60\right.$ e $\left.70{ }^{\circ} \mathrm{C}\right)$ e duas velocidades do ar de secagem $(0,9$ e $1,9 \mathrm{~m} / \mathrm{s})$. O experimento foi conduzido empregandose o delineamento inteiramente casualizado, em um esquema fatorial $(4 \times 2)$ com quatro repetições.

A extração do óleo essencial foi realizada no Laboratório de Química do Departamento de Estudos Básicos e Instrumentais da UESB, pelo método de arraste a vapor d'água, conforme recomendado por Martins (1998), sendo o tempo de arraste de $120 \mathrm{~min}$ para $20 \mathrm{~g}$ de massa de plantas seca, por amostra

Após a obtenção do hidrolato (água + óleo), o óleo essencial foi lavado três vezes com $50 \mathrm{~mL}$ de pentano para remoção da fase aquosa, em funil de separação de $125 \mathrm{~mL}$. A fase orgânica foi transferida para um funil contendo sulfato de magnésio anidro, em excesso. Posteriormente, o solvente foi removido em evaporador rotativo a temperatura de $40{ }^{\circ} \mathrm{C}$ até a redução expressiva do volume do solvente. Em seguida, o óleo foi transferido para um frasco âmbar, previamente pesado, para cálculo do rendimento do óleo.

As quantificações dos óleos essenciais, extraídos do manjericão, foram realizadas pela pesagem das amostras em balança analítica com precisão de 0,001 g. Após a pesagem os frascos foram fechados, lacrados com parafilme, envolvidos com papel alumínio e armazenados a $5^{\circ} \mathrm{C}$, até o momento da análise química do óleo.
A análise do óleo essencial foi realizada no Instituto de Química da Bahia, Laboratório de Pesquisa e Desenvolvimento em Química (UFBA). A quantificação do linalol foi determinada por cromatografia em fase gasosa, usando aparelho Shimadzu- QP 2010, equipado com detector de chama e coluna capilar HP-5MS $(30 \mathrm{~m} \times 1 \mathrm{~mm}$ x $0,25 \mathrm{~mm}$ ) com temperatura variando de 80 a $240{ }^{\circ} \mathrm{C}$. $\mathrm{O}$ gás de arraste utilizado foi o hélio, na vazão de $1 \mathrm{~mL} / \mathrm{min}$. A temperatura do injetor foi encurtida a $150{ }^{\circ} \mathrm{C}$.

Para a construção das curvas analíticas, foi preparada uma solução estoque do padrão de linalol, na concentração de 986,67, mg/mL diluída em dicloropentano. A solução estoque foi diluída de forma a obter as seguintes concentrações $1,2,5,8,10,50 \mathrm{mg} / \mathrm{mL}$.

\section{RESULTADOS E DISCUSSÃO}

Os teores médios de óleo essencial, obtidos em 20 g de folhas secas, são apresentados e em gramas para cada tratamento na Tabela 1.

TABELA 1 - Teores médios de óleos essenciais obtidos por arraste a vapor, para cada uma das temperaturas e vazões estudadas.

\begin{tabular}{|c|c|c|}
\hline $\begin{array}{c}\text { Temperatura } \\
{\left[{ }^{\circ} \mathrm{C}\right]}\end{array}$ & $\begin{array}{c}\text { Velocidade de } \\
\text { ar } \\
{[\mathrm{m} / \mathrm{s}]}\end{array}$ & $\begin{array}{c}\text { Teor de óleo } \\
\text { essencial } \\
{[\mathrm{g}] \times \mathbf{1 0}^{3}}\end{array}$ \\
\hline \multirow{2}{*}{40} & 0,9 & 122 \\
\hline & 1,9 & 196 \\
\hline \multirow{2}{*}{50} & 0,9 & 128 \\
\hline & 1,9 & 130 \\
\hline \multirow{2}{*}{60} & 0,9 & 92 \\
\hline & 1,9 & 94 \\
\hline \multirow{2}{*}{70} & 0,9 & 44 \\
\hline & 1,9 & 33 \\
\hline
\end{tabular}


A análise de variância mostrou o efeito tanto da temperatura como da velocidade ( $\mathrm{p}<0,01$, pelo teste de $\mathrm{F}$ ), bem como da interação entre elas, sobre a massa de óleo extraída. Como a interação foi significativa, procedeu-se à análise do efeito da temperatura, dentro de cada velocidade e da velocidade dentro de cada temperatura. Encontrou-se diferença significativa ( $\mathrm{p}<0,01$ pelo teste de Scheffé), na comparação de todas as temperaturas, entre si, dentro de cada velocidade e na comparação das duas velocidades em todas as temperaturas.

Para melhor discriminar o efeito da temperatura e da velocidade do ar, foi estabelecida uma equação de regressão linear múltipla, relacionando temperatura $\left({ }^{\circ} \mathrm{C}\right) \mathrm{e}$ velocidade do ar $(\mathrm{m} / \mathrm{s})$ com a massa de óleo bruto (Figura 2). As curvas apresentadas na Figura 2 foram obtidas com a equação 1 , para a qual o coeficiente de determinação $\left(\mathrm{R}^{2}\right)$ foi de 0,9632 .

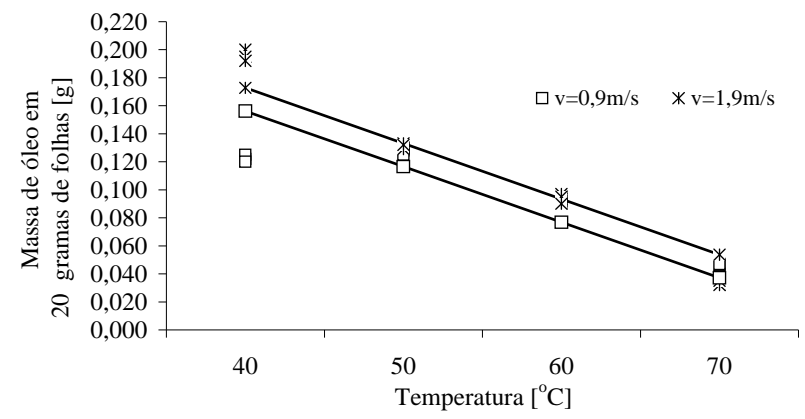

FIGURA 2 - Massa de óleo bruto em função da temperatura e da velocidade do ar de secagem.

O maior teor de óleo essencial foi obtido na temperatura de $40{ }^{\circ} \mathrm{C}$ diminuindo-se esse teor com o aumento da temperatura. Estes resultados são semelhantes aos obtidos por Deans \& Svoboda (1992), que também observaram a diminuição do teor de óleo essencial com aumento da temperatura do ar de secagem, quando empregaram temperaturas do ar de secagem entre 40 e 100 ${ }^{\circ} \mathrm{C}$ durante 24 horas, para a secagem de manjerona (Origanum majorana L.).

Na Tabela 2, estão relacionados os constituintes em maior quantidade identificados no óleo essencial do manjericão.

Uma equação de regressão não linear múltipla foi obtida, relacionando temperatura e velocidade com o teor de linalol (Figura 3). A Equação 2, obtida por análise de regressão apresentou $\mathrm{R}^{2}$ de 0,97 e a análise de variância da regressão confirmou, no nível de $1 \%$ de probabilidade, o efeito tanto da temperatura como da velocidade do ar de secagem, no teor de linalol.

TABELA 2 - Compostos em maior quantidade nas amostras provenientes dos tratamentos de secagem.

\begin{tabular}{cccc}
\hline Componente & $\begin{array}{c}\text { MM } \\
\text { [KDa] }\end{array}$ & $\begin{array}{c}\mathbf{t}_{\mathbf{R}} \\
{[\mathbf{m i n}]}\end{array}$ & $\begin{array}{c}\text { IK } \\
{[-]}\end{array}$ \\
\hline eucaliptol & 154 & 11,97 & 1005 \\
linalol & 154 & 14,40 & 1081 \\
eugenol & 164 & 23,72 & 1375 \\
ciclohexano & 204 & 25,08 & 1375 \\
a-bergamoteno & 204 & 26,43 & 1415 \\
não identificado & 204 & 29,16 & 1465 \\
naftaleno & 222 & 32,99 & 1699 \\
8-hexil-pentadecano & 250 & 41,30 & 1870 \\
\hline
\end{tabular}

$\left(\mathrm{MM}=\right.$ massa molar, $\mathrm{t}_{\mathrm{R}}=$ tempo de retenção, $\mathrm{IK}=$ Índice de Kovats)

A análise de variância da regressão confirmou, no nível de $1 \%$ de probabilidade, o efeito tanto da temperatura como da velocidade do ar estudados.

O componente majoritário (linalol) apresentou os maiores rendimentos nas temperaturas de 50 e $60{ }^{\circ} \mathrm{C}$, resultados semelhantes aos obtidos por Pereira et al. (2000) que obtiveram maior rendimento de cumarina nas folhas de guaco (Mikania glomerata Sprengel) secas a $50{ }^{\circ} \mathrm{C}$, concluindo que o conteúdo de cumarina é afetado pela temperatura de secagem.

Uma análise de pontos críticos da equação (2) obtida permitiu deduzir que, assumindo-se a validade de uma interpolação dos dados, o teor máximo de linalol, seria obtido na velocidade de $1,9 \mathrm{~m} / \mathrm{s}$, com uma temperatura de $54,4{ }^{\circ} \mathrm{C}$. Estes resultados estão de acordo com os encontrados por Blanco et al. (2002a,b), os quais demonstraram que temperaturas de secagem igual ou superior a $40{ }^{\circ} \mathrm{C}$, para a Mentha piperita L. (hortelã) e Rosmarinus officinalis L. (alecrim), causaram alterações significativas nos constituintes de seus óleos essenciais. Segundo Randuz et al. (2003), o aumento ou a redução dos compostos pode ser ocasionado por reações de oxidação, redução e rearranjos durante o processo de secagem devido à temperatura ou ao longo do tempo de secagem.

Nas amostras submetidas à temperatura de $70^{\circ} \mathrm{C}$ observou-se que todos os constituintes sofreram grandes reduções em seus teores, indicando, provavelmente, perdas por volatilização. 
$\mathrm{L}=-0,0089 \mathrm{~T}^{2}+0,9737 \mathrm{~T}+1,8552 \mathrm{~V}^{2}-4,7755 \mathrm{~V}-21,7263$

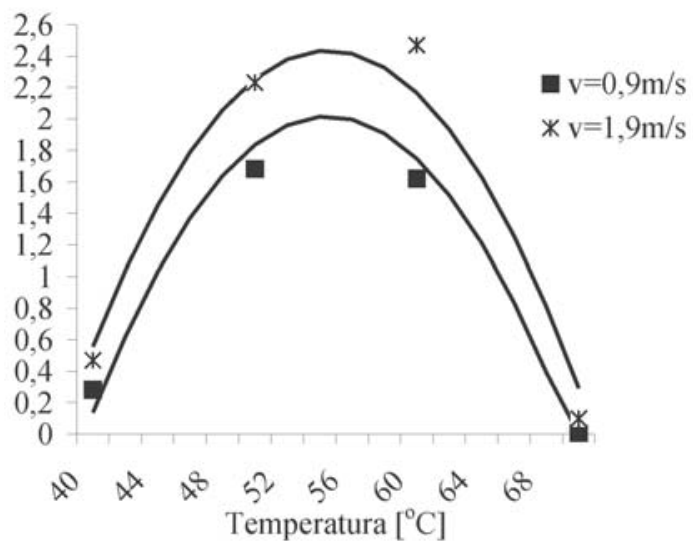

FIGURA 3 - Teor de linalol no óleo bruto em função da velocidade e da temperatura de secagem.

Analisando os resultados obtidos com CG/MS, observou-se que, apesar da temperatura de $40{ }^{\circ} \mathrm{C}$ ter fornecido maior quantidade de óleo essencial, a quantidade de compostos foi fortemente afetada pelo tempo de exposição das folhas ao ar de secagem, o que promoveu uma redução nos teores dos compostos químicos. Novamente, este resultado pode, provavelmente, ser atribuído à volatilização.

\section{CONCLUSÕES}

Os maiores rendimentos extrativos de óleos essenciais de manjericão foram obtidos quando o processo de secagem foi realizado com temperatura do ar igual a 40 ${ }^{\circ} \mathrm{C}$. Contudo, o maior rendimento de linalol foi obtido quando o processo de secagem foi realizado com temperatura do ar na faixa de 50 a $60{ }^{\circ} \mathrm{C}$.

A composição química do óleo essencial do manjericão foi afetada pelos variáveis da secagem tanto pela temperatura como pela velocidade do ar de secagem. Para as condições deste experimento, recomenda-se que a temperatura e a velocidade do ar para secagem de manjericão, visando obter maior teor de óleo essencial e a maior concentração de linalol, sejam de $54,4{ }^{\circ} \mathrm{C}$ e $1,9 \mathrm{~m} / \mathrm{s}$ respectivamente.

\section{REFERÊNCIAS BIBLIOGRÁFICAS}

ASAE STANDARDS. Standards engineering practices: data: moisture measurement-forages, ASAE S358.2 DEC99. American Society of Agricultural Engineers, [S.1.], p. 565$572,2000$.
BARITAUX, O.; RICHARD, T. J.; DERBESY, M. Effects of drying and storage of herbs and spices on the essential oil: part 1: Basil, Ocimum basilicum L. Flavour and Fragance Journal, [S.1.], v. 7, p. 267-271, 1992.

BLANCO, M. C. S. G.; MING, L. C.; MARQUES, M. O. M.; BOVI, O. A. Drying temperature effects in peppermint essential oil content and composition. Acta Horticulturae, Amsterdam, n. 569, p. 95-98, 2002a.

BLANCO, M. C. S. G.; MING, L. C.; MARQUES, M. O. M.; BOVI, O. A. Drying temperature effects in rosemary essential oil content and composition. Acta Horticulturae, Amsterdam, n. 569, p. 99-103, 2002b.

CHARLES, D. J.; SIMON, J. E. Comparison of extraction methods for the rapid determination of essential oil content and composition of basil. Journal of the American Society for Horticultural Science, Alexandria, v. 115, n. 3, p. 458462,1990 .

DEANS, S. G.; SVOBODA, K. P. Effects of drying regime on volatile oil and microflora of aromatic plants. Acta Horticulturae, Amsterdam, n. 306, p. 450452, 1992.

HANSEN, R. C.; KEENER, H. M.; ELSOHLY, H. N. Thin layer drying of cultivated taxus clippings. Transactions of the ASAE, Madison, v. 36, n. 5, p. 1387-1391, 1993.

JORGE, L. I. F.; ROQUE, N. F.; FERRO, V. O. Ocimum micranthum Willd: manjericão do Brasil: caracterizações histólogica e química. Revista do Instituto Adolfo Lutz, São Paulo, v. 52, n. 1/2, p. 47-50, 1992.

KAMADA, T.; CASALI, V. W. D.; BARBOSA, L. C. A.; FORTES, I. C. P.; FINGER, F. L. Plasticidade fenotípica do óleo essencial em acessos de manjericão (Ocimum spp.). Revista Brasileira de Plantas Medicinais, São Paulo, v. 1, n. 2, p. 13-22, 1999.

MARTINS, E. R. Estudos em Ocimum selloi Behtn.: isozimas, morfologia e óleo essencial. In: LIN CHAU MING (Org.). Plantas medicinais, aromáticas e condimentares: avanços na pesquisa agronômica. Botucatu: UNESP, 1998. v. 2, p. 97-125.

MARTINS, E. R.; CASTRO, D. M.; CASTELLANI, D. C.; DIAS, J. E. Plantas medicinais. Viçosa: UFV, 1994. $220 \mathrm{p}$. 
MARTINS, P. M. Influência da temperatura e velocidade do ar de secagem no teor e na composição química do óleo essencial de capim limão (Cymbopogum citratus (D.C.) STAPF). 2000. 77 f. Dissertação (Mestrado em Engenharia Agrícola) - Universidade Federal de Viçosa, Viçosa, 2000.

MÜLLER, J.; MÜHLBAUER, W. Effects of drying on the essential oil of Chamomile recutita. In: INTERNATIONAL JOINT SYMPOSIUM OF BIOLOGYAND CHEMISTRY OF ACTIVE NATURAL SUBSTANCES, 1992, Bonn. Anais... Bonn: [s.n.], 1992.

NTEZURUBANZA, L.; SHEFFER, J. J. C.; LOOMAN, A. Composition of essential oil of Ocimum kilimandscharicum grown in Ruanda. Planta Medica, [S.1.], v. 50, n. 5, p. 385388, 1984.

PEREIRA, A. M. S.; CÂMARA, F. L. A.; CELEGHINI, R. M. S.; VILEGAS, J. H. Y.; LANÇAS, F. M.; FRANÇA, S. C. Seasonal variation in coumarin content of Mikania glomerata. Journal of Herbs, Spices e Medicinal Plants, [S.1.], v. 7, n. 2, p. 1-10, 2000.

PEREZ, A. M. J.; VELASCO, N. A.; DURU, M. E. Composition of the essential oils of Ocimum basilicum var. glabratum and Rosmarinus officinalis from Turkey. Journal of Essential Oil Research, [S.1.], v. 7, n. 1, p. 7375,1995 .
RADÜNZ, L. L.; MELO, E. C.; BERBERT, P. A.; ALMEIDA, L. C. B.; ROCHA, R. P.; GRANDI, A. M. Efeitos da temperatura do ar de secagem sobre a qualidade do óleo essencial de alecrim pimenta (Lippis sidoides Cham). Revista Brasileira de Armazenamento, Viçosa, v. 27, n. 2, p. 9-13, 2002.

RADÜNZ, L. L.; MELO, E. C.; BERBERT, P. A.; BARBOSA, L. C. A.; SANTOS, R. H. S.; ROCHA, R. P. Influência da temperatura do ar de secagem na quantidade do óleo essencial extraído de guaco (Mikania glomerata Sprengel). Revista Brasileira de Armazenamento, Viçosa, v. 28, n. 2, p. 41-45, 2003.

TEIXEIRA, J. P. F.; MARQUES, M. O. M.; FURLAN, P. R.; FACANALLI, R. Óleo essencial de duas variedades de manjericão em cultivo hidropônico. Horticultura Brasileira, Brasília, v. 18, p. 982-983, 2000. Suplemento.

VENSKUTONIS, P. R. Effect of drying on the volatile constituents of thyme (Thymus vulgaris L.) and sage (Salvia officinalis, L.). Food Chemistry, Washington, v. 59, n. 2, p. 219-227, 1997.

VENSKUTONIS, P. R.; POLL, L.; LARSEN, M. Influence of drying and irradiantion on the composition of volatile compounds of thyme (Thymus vulgaris L). Flavour and Fragance Journal, [S.1.], v. 11, p. 123-128, 1996. 